\title{
PROOF OF A CONJECTURE OF ASKEY ON ORTHOGONAL EXPANSIONS WITH POSITIVE COEFFICIENTS
}

BY WILLIAM F. TRENCH

Communicated by Walter Gautschi, May 22, 1975 [2].

We prove the following theorem, which was conjectured by R. Askey [1] ,

THEOREM 1. Let $\left\{p_{n}(x)\right\}$ and $\left\{q_{n}(x)\right\}$ be polynomials orthogonal over $[0, \infty)$ with respect to distributions $d u(x)$ and $x^{c} d u(x)(c>0)$, respectively, normalized so that

$$
p_{i}(0)>0, \quad q_{i}(0)>0, \quad i=0,1, \ldots
$$

Then the coefficients $\left\{a_{j n}\right\}$ in the expansions

$$
q_{n}(x)=\sum_{j=0}^{n} a_{j n} p_{j}(x), \quad n=0,1, \ldots
$$

are all positive.

It is to be understood here that $d u(x)$ has moments of all orders on $[0, \infty)$, and that $n \leqslant N-1$ if $d u(x)$ is a discrete distribution over only $N$ points.

Using different arguments, Askey [1] and the author [4] have shown that the conclusion of this theorem follows from classical results if $c$ is a positive integer, and, allowing for a difference in normalization, results obtained in [4] imply also that the coefficients in the "inverse" expansion, $p_{n}(x)=$ $\sum_{j=0}^{n} b_{j n} q_{j}(x)$, satisfy $(-1)^{n-i} b_{j n} \geqslant 0$ if $c$ is a positive integer, but that this does not hold for all positive $c$.

It suffices to prove Theorem 1 for $0<c<1$, since its conclusion follows for any positive $c$ from finitely many successive applications of this restricted result. We confine our attention to this case.

The roots of $p_{i}(x)$ and $q_{i}(x)$ are positive; therefore, (1) and Descartes' rule of signs imply that

$$
(-1)^{r} p_{i}^{(r)}(0) \geqslant 0, \quad(-1)^{r} q_{i}^{(r)}(0) \geqslant 0, \quad 0 \leqslant r \leqslant i .
$$

This is needed below.

LEMMA. Under the assumptions of Theorem 1,

$$
(-1)^{j} a(a-1) \cdots(a-j+1) \int_{0}^{\infty} x^{a} p_{j}(x) d u(x)>0
$$


if $j \geqslant 1, a \geqslant 0$, and $a \neq 0,1, \ldots, j-1$.

Proof. Let $h(x)$ be the projection of $x^{a}$ on the space of polynomials of degree $\leqslant j$, with respect to the inner product

$$
(f, g)=\int_{0}^{\infty} f(x) g(x) d u(x)
$$

thus,

$$
h(x)=b_{0} p_{0}(x)+\cdots+b_{j} p_{j}(x)
$$

where

$$
b_{k}=\frac{1}{\left\|p_{k}\right\|^{2}} \int_{0}^{\infty} x^{a} p_{k}(x) d u(x), \quad k=0, \ldots, j
$$

Since $x^{a}-h(x)$ is orthogonal to every polynomial of degree $\leqslant j$, it has at least $j+1$ zeros on $(0, \infty)$, and so its $j$ th derivative has at least one. Therefore,

$$
a(a-1) \cdots(a-j+1) x_{1}^{a-j}-b_{j} p_{j}^{(j)}(0)=0
$$

for some positive $x_{1}$, and (4) follows from this and (5) (with $k=j$ ), since $(-1)^{j} p_{j}^{(j)}(0)>0$.

Proof of Theorem 1. From (4),

$$
(-1)^{r} \int_{0}^{\infty} x^{r+c} p_{j}(x) d u(x)<0, \quad 0 \leqslant r<j, \quad 0<c<1 .
$$

Since

$$
\int_{0}^{\infty} p_{i}(x) p_{j}(x) x^{c} d u(x)=\sum_{r=0}^{i} \frac{p_{i}^{(r)}(0)}{r !} \int_{0}^{\infty} x^{r+c} p_{j}(x) d u(x),
$$

(3) and (6) imply that

$$
\int_{0}^{\infty} p_{i}(x) p_{j}(x) x^{c} d u(x)<0, \quad 0 \leqslant i<j, \quad 0<c<1,
$$

and this inequality also holds for $j<i$, by symmetry.

Now we see from (2) that $\left(a_{0 n}, \ldots, a_{n n}\right)$ is the solution of the system

(8) $\int_{0}^{\infty} p_{i}(x) q_{n}(x) x^{c} d u(x)=\sum_{j=0}^{n} a_{j n} \int_{0}^{\infty} p_{i}(x) p_{j}(x) x^{c} d u(x), \quad 0 \leqslant i \leqslant n$,

which has a symmetric, positive definite matrix $H$ with negative off-diagonal elements (cf. (7)). Stieltjes [3] (see also [5, §3.5]) showed that every element in the inverse of such a matrix is positive. As for the left side of (8), the orthogonality of $\left\{q_{r}(x)\right\}$ with respect to $x^{c} d u(x)$ implies that

$$
\int_{0}^{\infty} p_{i}(x) q_{n}(x) x^{c} d u(x)=\delta_{i n} \frac{p_{n}^{(n)}(0)}{q_{n}^{(n)}(0)} \int_{0}^{\infty}\left(q_{n}(x)\right)^{2} x^{c} d u(x), \quad 0 \leqslant i \leqslant n ;
$$


since $p_{n}^{(n)}(0) / q_{n}^{(n)}(0)>0$ (from (3)), it now follows from the positivity of $H^{-1}$ that $a_{r n}>0(0 \leqslant r \leqslant n)$. This completes the proof of Theorem 1 .

The idea of applying Stieltjes' theorem here came from a paper by $M$. W. Wilson [6]; the last paragraph of our proof is essentially due to him.

Other general theorems on positivity (or nonnegativity) of the coefficients in (2) can be obtained by arguments related to the one used here. These results will be published elsewhere.

\section{REFERENCES}

1. R. Askey, Orthogonal expansions with positive coefficients, Proc. Amer. Math. Soc. 16 (1965), $1191-1194 . \quad$ MR 32 \#2799.

2. Orthogonal polynomials and positivity, Studies in Appl. Math., no. 6, SIAM, Philadelphia, Pa., 1970.

3. T. J. Stieltjes, Sur les racines de l'equation $X_{n}=0$, Acta Math. 9 (1887), 385-400.

4. W. F. Trench, Nonnegative and alternating expansions of one set of orthogonal polynomials in terms of another, SIAM J. Math. Anal. 4 (1973), 111-115. MR 48 \#4628.

5. R. S. Varga, Matrix iterative analysis, Prentice-Hall, Englewood Cliffs, N. J., 1962. MR $28 \# 1725$.

6. M. W. Wilson, Nonnegative expansions of polynomials, Proc. Amer. Math. Soc. 24 (1970), 100-102. MR 44 \#451.

DEPARTMENT OF MATHEMATICS, DREXEL UNIVERSITY, PHILADELPHIA, PENNSYLVANIA 19104 